\title{
The effectiveness of providing evidenced-based perinatal practice to low-income populations providing perinatal care: Does patient income influence the delivery of quality care?
}

\author{
Amy L. Damon ${ }^{1}$, Carmen D. Parrotta ${ }^{2}$, Lindsey A. Wallace ${ }^{3}$, William Riley ${ }^{4}$ \\ 1. Economics Department, Macalester College, St. Paul, Minnesota, USA. 2. Quality and Innovations Department, Fairview \\ Medical Group, Minneapolis, Minnesota, USA. 3. School of Public Health, University of Minnesota, Minneapolis, Minnesota, \\ USA. 4. School for the Science of Health Care Delivery, Arizona State University, Phoenix, Arizona, USA
}

Correspondence: Amy L. Damon. Address: Economics Department, Macalester College, St. Paul, Minnesota, USA. E-mail: adamon@macalester.edu

Received: February 25, 2013

DOI: $10.5430 /$ jha.v2n4p82

\section{Abstract}

Background: This study examines a national perinatal quality improvement collaborative designed to create high reliability through the use of evidence-based perinatal care bundles. The objective of this study is to determine whether hospitals serving low-income patient populations experienced lower compliance with perinatal care bundles than hospitals serving higher-income patient populations.

Objective: We investigated the relationship between the rate of perinatal bundle compliance within a hospital and the economic characteristics of the patients and surrounding community. We hypothesized a negative relationship between poverty and care bundle compliance.

Methods: Using prospective data from 131,847 births over 34 months within 16 hospitals located in cities across the United States, we examined the relationship between compliance with evidence-based obstetrical care bundles and three measures of the poverty status of the patient population served and the hospital service area: 1) proportion of the obstetrical patients with Medicaid as the primary payer, 2) median income in the hospital service area, and 3) poverty rate in the hospital's service area.

Results: The findings indicate no difference in bundle compliance rates in relation to the economic characteristics of the participating hospitals and their patients.

Conclusions: While previous research has indicated that patients of lower socioeconomic status are less likely to receive high quality care, the findings in this study indicate that hospital compliance with evidence-based perinatal care bundles did not differ by economic characteristics of the hospital service area. These results indicate uniformity of care across hospitals irrespective of patient economic characteristics.

\section{Key words}

Health care quality, Perinatal care, Bundle compliance, Hospital service area poverty, Socioeconomic status 


\section{I ntroduction}

Maternal and neonatal care represents the greatest portion of Medicaid's hospitalization costs $(27 \%)^{[1]}$. In 2009 , there were more than 4.1 million births in the U.S ${ }^{[2]}$. Considering these two statistics, it's critical that the healthcare delivery system provides high-quality perinatal care to better control rising costs for a growing population. In addition, numerous studies have documented persistent racial, ethnic, and socioeconomic disparities in maternal morbidity and mortality, preterm births, low birth weight infants, and other adverse outcomes ${ }^{[3-5]}$. Black women are nearly four times more likely to die from pregnancy-related causes than White women ${ }^{[6]}$. Individuals of low socioeconomic status are less likely to receive high quality care in hospitals ${ }^{[7]}$. Results of the 2005 National Healthcare Quality and Disparities Report concluded that low-income patients experience low quality care for approximately two-thirds of the quality measures included for analysis ${ }^{[8]}$. Based on existing studies it appears evident that being economically disadvantaged leads to similarly disadvantaged healthcare services. Furthermore, being socioeconomically disadvantaged is linked with poor health throughout the lifecycle, from birth, through childhood and adolescence, and into adulthood and old age ${ }^{[9]}$ and greater income disparity is linked with higher rates of perinatal morbidity/mortality ${ }^{[10]}$.

This study analyzes results from a multi-site hospital quality improvement initiative that aims to increase the reliability of obstetric care services. It explores the relationship between hospitals serving lower income communities and their ability to achieve high reliability in patient safety. Prior efforts to improve reliability have generated positive contributions to care processes for a variety of clinical conditions including ventilator acquired pneumonia ${ }^{[1]}$, central line blood stream infections ${ }^{[12]}$, and perinatal care ${ }^{[13]}$. Research suggests that cesarean delivery and elective induction associated with pregnancy and childbirth can be reduced through adherence to existing evidence-based guidelines ${ }^{[14]}$. However, there have been no investigations into the extent to which improved care process performance is related to characteristics of the hospital's patient population or service area, especially the level of poverty. In this study, we report a systematic effort to improve high reliability in the perinatal units of sixteen hospitals across the nation. We then examine the relationship between obstetrical unit reliability and measures of poverty in the hospital services area and among its patient population.

Due to the evidence that poverty and low socioeconomic status are linked with poor health care outcomes, including poor perinatal health outcomes, our team hypothesized that hospitals serving higher income patient populations would have significantly higher reliability.

\section{Materials and methods}

\subsection{Study design}

This prospective observational study explores the relationship between the extent of adoption of perinatal best practices and poverty measures reflecting the hospital patient care mix. It is part of a larger study to reduce perinatal harm by introducing three evidence-based practices (elective induction bundle, augmentation bundle, and vacuum bundle) into the perinatal units of hospitals participating in the study. The design of this study is to introduce bundle compliance training which focuses on three distinct elements of standardizing perinatal care: augmentation, elective induction, and vacuum or forceps delivery.

\subsection{Setting}

The intervention group consisted of 16 hospitals across the U.S. that volunteered to participate in a collaborative to improve perinatal care processes and outcomes. The 16 hospitals in the study account for approximately 47,000 births per year for a total of 131,847 deliveries during the study period. The hospitals were located across the United States in the following cities: Akron, OH; West Allis, WI; Louisville, KY; Springfield, MA; Cincinnati, OH (two hospitals); Burnsville, MN; Fort Worth, TX; Kingsport, TN; Peoria, IL; Phoenix, AZ; Martinsburg, WV; Dallas, TX; Albuquerque, NM; Chewelah, WA; and Minneapolis, MN. 


\subsection{Intervention}

A care bundle is a group of interventions based on best available evidence, published best practices, and national standards established by leading professional health care associations, which must be performed in coordination with each other ${ }^{[15]}$. The selection of the evidence-based elements constituting the bundles is based on sound science, and an agreement among clinicians that patients should receive all elements of care unless medically contraindicated ${ }^{[16]}$. Application of bundles to health care processes is a technique to reduce variability, an important part of achieving high reliability ${ }^{[17]}$.

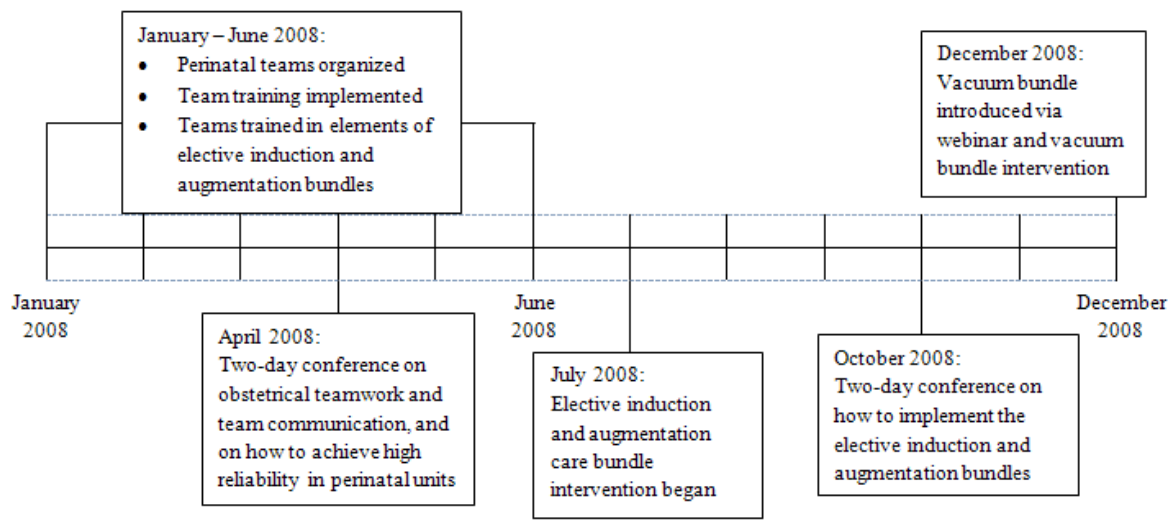

Figure 1. Implementation Timeline for Perinatal Safety Initiative

The intervention was conducted over a 36-month period from January, 2008 to December, 2010. In the initial six months training protocols were developed, perinatal teams at each intervention hospital were organized, and team training was initiated. Figure 1 shows the implementation timeline for the intervention. Team training consisted of educating teams about the elements of each of the three care bundles described in Table 1. Several quality improvement models have been successfully used to improve reliability ${ }^{[18]}$, and the three interventions in this initiative focus on the areas of highest risk for obstetrical harm ${ }^{[19]}$. Three bundles were adopted from prior studies: 1) elective induction bundle, 2) augmentation bundle, and 3) vacuum bundle ${ }^{[20,21]}$.

Table 1. Bundles and Bundle Components

\begin{tabular}{lll}
\hline Elective Induction & Augmentation & Vacuum \\
\hline Gestational age $\geq 39$ weeks & $\begin{array}{l}\text { Documentation of estimated } \\
\text { fetal weight }\end{array}$ & $\begin{array}{l}\text { Alternative labor strategies considered } \\
\text { Patient prepared }\end{array}$ \\
$\begin{array}{l}\text { Normal Fetal status (per NICHD tiers) prior to } \\
\text { onset of Oxytocin }\end{array}$ & $\begin{array}{l}\text { NICHD tiers) } \\
\text { Pelvic exam prior to the onset of Oxytocin }\end{array}$ & $\begin{array}{l}\text { Pelvic exam prior to the onset } \\
\text { of Oxytocin }\end{array}$ \\
$\begin{array}{l}\text { Recognition and management of tachysystole } \\
\text { Recognition and management } \\
\text { of tachysystole }\end{array}$ & $\begin{array}{l}\text { Maximum application time and } \# \text { of pop-offs } \\
\text { predetermined and documented } \\
\text { delivery }\end{array}$ \\
& &
\end{tabular}

\subsection{Data sources and variable measurement}

We identified three measures of poverty and three care process measures for this study. Poverty in the hospital service areas and patient populations were measured using three metrics: 1) the proportion of deliveries paid by Medicaid, 2) the median income level in the hospital service area, and 3) the proportion of poverty in the hospital service area. These three measurements of poverty are used as independent explanatory variables in the descriptive analyses presented. Data for 
these poverty-related metrics were collected from three sources. The proportion of Medicaid deliveries was collected through chart reviews provided by key personnel within the obstetrical units or collected by finance staff using administrative data within each study hospital. The median income level and proportion of poverty in a hospital service area were determined by querying the Dartmouth Atlas ${ }^{[22]}$ database for descriptions of hospital service areas by zip codes. These zip codes were then identified on the U.S. Census Bureau website for average income and poverty level. The median income and percentage of poverty measure the service area while the Medicaid metric measures patients.

Three process measures of care quality were used in this study: augmentation bundle, elective induction bundle, and vacuum bundle compliance. Table 1 shows the three obstetric care bundles and the behavior-specific interventions related to each bundle. Bundles are collections of processes needed to care effectively and safely for patients undergoing particular treatments with inherent risks ${ }^{[15]}$ and have been extensively studied in perinatal settings ${ }^{[23-24]}$. Each care bundle consists of four to five evidence-based clinical care elements in order to standardize clinical processes and reduce variation in practice.

The elective induction bundle is designed to eliminate neonatal harm from the early induction of labor without medical indication ${ }^{[15]}$. This care bundle requires that the fetus is at least 39 weeks gestation to prevent harm from premature delivery, the fetal heart rate monitoring to document stable fetal status, pelvic examination to determine the readiness of the cervix to give birth, and defining a plan to deal with the potential for tachysystole (uterine hyperstimulation) which occurs when contractions become too close together ${ }^{[15,21]}$. The augmentation bundle was developed to reduce the unnecessary use of oxytocin during delivery which can lead to potential complications. This care bundle requires the physician to assess and document the estimated fetal weight, monitor fetal heart rate to document stable fetal status, conduct pelvic assessment exam, and monitor tachysystole ${ }^{[15,21]}$. The vacuum bundle was designed to standardize care for operative vaginal delivery to optimize maternal and neonatal outcomes. This care bundle requires the clinician to discuss and consider alternative labor strategies with the patient, the patient is prepared to undergo a vacuum-assisted delivery and both the patient and clinician to feel the procedure has a high probability of success. The vacuum bundle also requires predetermining maximum application time and number of re-applications as well as the availability of cesarean and resuscitation teams.

All elements of a care bundle must be met for participating hospitals to receive credit for compliance, as evidenced in the monthly chart audits. For each patient, a bundle is scored as an "all or none" measure. Each of the clinical elements for the respective bundle is coded yes or no for whether it was performed for each patient. All items for each bundle are required to be present for an observed event in order for a delivery to qualify as bundle compliant.

Data were collected on each bundle element to help teams identify areas for improvement in order to achieve overall bundle compliance. Bundle compliance is measured using a chart audit. A chart audit is simply a review of patients' charts to determine if the appropriate care bundle was implemented in each case. A data collection protocol was distributed to each hospital describing the procedures for chart audits, the metrics for each bundle, and a process to upload data on a monthly basis. The protocol required: 1) random selection of 20 elective induction charts, 20 augmentation charts, and 20 vacuum delivery charts each month; 2) detailed review of each randomly selected chart to ensure all elements of each bundle had been addressed; and 3) notation of compliance with appropriate steps taken to address documented patient conditions (for example, if tachysystole is documented it is noted whether the team followed the policy algorithm for responding to tachysystole).

Data entry was completed by designated individuals in each obstetrics team every month for the three care bundles, with additional birth $\log$ data entered on a monthly basis. These monthly observations of 20 expected audits were then aggregated into monthly compliance rates, with "yes" $=100 \%$ compliance and "no" $=0 \%$ compliance. For a given bundle, monthly audits of randomly selected hospital records yielded a compliance measure for each bundle element achieved, and monthly entries over all metric categories expected to be 60 audits per month, per team. If a hospital did not have at least 
20 deliveries in a given month for a bundle audit to be deemed adequate, a 100\% chart audit was conducted to ensure compliance with the respective bundle. Each hospital compliance rate was compiled over the three year study period for each bundle compliance measure and each poverty measure. The bundle compliance rates for the 16 hospitals are based on an average of approximately 3,250 deliveries in each hospital per year over the total study period.

Table 2. Profile of Intervention Group Hospitals

\begin{tabular}{lllll}
\hline Hospital Characteristics (N=16) & Mean & Standard Deviation & Minimum & Maximum \\
\hline Births per year & 3,248 & 1,697 & 912 & 6,655 \\
Total beds per hospital & 430 & 232 & 149 & 898 \\
Academic (vs community) hospital & $31 \%$ & - & 0 & 1 \\
Population in service area & 783,513 & 700,108 & 8,463 & $2,168,299$ \\
Geographic region & & & & \\
$\quad$ Midwest & $44 \%$ & - & 0 & 1 \\
$\quad$ Northeast & $6 \%$ & - & 0 & 1 \\
$\quad$ South & $19 \%$ & - & 0 & 1 \\
$\quad$ Southwest & $6 \% \%$ & - & 0 & 1 \\
$\quad$ West & & - & 0 & 1 \\
Key poverty measures & $\$ 44,762$ & $\$ 8,191$ & & \\
$\quad$ Median income in service area & $9 \%$ & $3 \%$ & $\$ 32,117$ & $\$ 66,975$ \\
Poverty rate in service area & $38 \%$ & $16 \%$ & $2 \%$ & $13 \%$ \\
Percent of patients with Medicaid coverage & & $14 \%$ & $62 \%$ \\
\hline
\end{tabular}

In order to determine the effect of our three poverty measures on bundle compliance included we used STATA to determine quartiles for percent Medicaid patients served, median income, and poverty in hospital service area. This data was examined against bundle compliance data to determine whether compliance rates differed by poverty measure.

\subsection{Statistical analysis}

We conducted both a descriptive analysis and a multivariate analysis of the data. The descriptive analysis examined each care bundle categorized by Medicaid patient percentage by quartile, median income by quartile, and percentage poverty by quartile in the hospital service area. The upper income quartile includes those in 76th - 100th percentile, the next includes 51 st -75 th percentile, the lower middle includes 26 th -50 th percentiles and the lowest group is the $0-25$ th percentile. These groupings were used to examine the mean compliance rate for each bundle across income groups.

We also conducted a set of multivariate ordinary least squares (OLS) regressions to explain each of the three care bundles by poverty measures. None of the relationships examined in the multivariate analysis were found to be statistically significant (although some did approach statistical significance), likely due to small sample size, so they are not covered in this paper.

\section{Results}

A descriptive analysis of selected characteristics of the 16 hospitals and the key poverty measures is summarized in Table 2. On average hospitals in this study have 3,248 births per year, ranging from 912 to 6,655 . Furthermore, $31 \%$ of the hospitals included are academic hospitals while the rest are community hospitals. Hospitals in this study have an average of 430 beds and all are in urban settings. The defined service area population ranges widely from approximately 8,400 people to over 2.1 million people. The average median income is $\$ 44,762$ with a standard deviation of $\$ 8,191$. Poverty rates also vary considerably from $2 \%$ to $13 \%$ with an average of $9 \%$ poverty in the hospital service areas. The percent of deliveries paid for by Medicaid is on average 38\% and ranges from $14 \%$ to $62 \%$. 
Table 3. Bundle compliance by median hospital service area Medicaid status (by quartiles) for collaborative hospital service areas

\begin{tabular}{lllllll}
\hline & $\mathbf{0 - 2 5 \%}$ & $\mathbf{2 5 \% - 5 0 \%}$ & $\mathbf{5 0 \% - 7 5 \%}$ & $\mathbf{7 5 \% - 1 0 0 \%}$ & Overall & $\boldsymbol{p}$-value \\
\hline $\mathrm{N}$ & 4 & 3 & 2 & 4 & 13 & \\
Average Medicaid & $19.63 \%$ & $35.00 \%$ & $45.16 \%$ & $54.81 \%$ & $37.93 \%$ & \\
Augmentation Compliance & $44.50 \%$ & $64.14 \%$ & $69.31 \%$ & $61.34 \%$ & $59.7 \%$ & 0.2505 \\
Induction Compliance & $73.28 \%$ & $69.26 \%$ & $82.33 \%$ & $81.28 \%$ & $76.3 \%$ & 0.167 \\
Vacuum Compliance & $35.78 \%$ & $47.73 \%$ & $37.49 \%$ & $45.00 \%$ & $41.0 \%$ & 0.8417 \\
\hline
\end{tabular}

Tables 3 through 5 show overall compliance for each bundle by quartile and one measure of poverty status; Medicaid patient status, median hospital service area income, and median hospital service area poverty level respectively.

Table 4. Bundle compliance by median hospital service area income (by quartiles) for collaborative hospital service areas

\begin{tabular}{lllllll}
\hline & $\mathbf{0 - 2 5 \%}$ & $\mathbf{2 5 \% - 5 0 \%}$ & $\mathbf{5 0 \% - 7 5 \%}$ & $\mathbf{7 5 \% - 1 0 0 \%}$ & Overall & $\boldsymbol{p}$-value \\
\hline $\mathrm{N}$ & 4 & 4 & 3 & 5 & 16 & \\
Average Income & $\$ 36,416$ & $\$ 42,226$ & $\$ 45,138$ & $\$ 53,242$ & $\$ 44,762$ & \\
Augmentation Compliance & $67.2 \%$ & $63.1 \%$ & $63.8 \%$ & $48.5 \%$ & $59.7 \%$ & 0.2582 \\
Induction Compliance & $76.3 \%$ & $73.8 \%$ & $81.7 \%$ & $75.1 \%$ & $76.3 \%$ & 0.767 \\
Vacuum Compliance & $43.7 \%$ & $43.1 \%$ & $51.5 \%$ & $31.0 \%$ & $41.0 \%$ & 0.4109 \\
\hline
\end{tabular}

Overall, hospitals achieved a $60 \%$ compliance with the augmentation bundle, $76 \%$ compliance with the induction bundle, and $41 \%$ compliance with the vacuum bundle. For all three care bundles, hospitals that serve areas with lower income demonstrate the same bundle compliance compared with hospitals that serve areas with higher income. The average compliance rates are not significantly different across income group quartiles, as shown by the $p$-values in the last column of Tables 3-5.

Table 5. Bundle compliance by median hospital service area poverty level (by quartiles) for collaborative hospital service areas

\begin{tabular}{lllllll}
\hline & $\mathbf{0 - 2 5 \%}$ & $\mathbf{2 5 \% - 5 0 \%}$ & $\mathbf{5 0 \% - 7 5 \%}$ & $\mathbf{7 5 \% - 1 0 0 \%}$ & Overall & $\boldsymbol{p}$-value \\
\hline $\mathrm{N}$ & 5 & 3 & 4 & 4 & 16 & \\
Average Poverty & $5.54 \%$ & $8.45 \%$ & $10.26 \%$ & $12.21 \%$ & $8.93 \%$ & \\
Augmentation Compliance & $48.48 \%$ & $65.52 \%$ & $60.04 \%$ & $68.93 \%$ & $59.7 \%$ & 0.1986 \\
Induction Compliance & $75.09 \%$ & $81.90 \%$ & $80.75 \%$ & $69.25 \%$ & $76.3 \%$ & 0.2473 \\
Vacuum Compliance & $31.00 \%$ & $49.20 \%$ & $43.66 \%$ & $44.85 \%$ & $41.0 \%$ & 0.4641 \\
\hline
\end{tabular}

Although the multivariate regression is not discussed in detail in this paper it is worthwhile to mention a few relationships that bordered on statistically significant; augmentation bundle compliance and percent poverty in service area had a positive relationship $(p=0.064)$, augmentation bundle compliance and median income in hospital service area had a negative relationship $(p=0.098)$, induction bundle compliance and percent Medicaid patients had a positive relationship $(p=0.138)$, and vacuum bundle compliance and median income in hospital service area had a negative relationship $(p=0.054)$. 


\section{Discussion}

Despite the fact that we generally think high quality care is only accessible within hospitals that serve high income populations, our findings demonstrate that this may not be true in the case of the perinatal safety initiative. There was no strong socioeconomic difference that we could observe among our sample when it came to bundle compliance, and weak evidence that hospitals in poorer areas are doing a better job at bundle compliance than those in wealthier areas or with wealthier patient populations.

These findings suggest that regardless of the socioeconomic characteristics of patient population, the 16 hospitals on our study provided consistent quality of care across the board. These results indicate that hospitals serving low-income populations are administering high quality care, despite barriers to doing so. The use of bundles in healthcare is a relatively new phenomenon; these results indicate that bundles may be a healthcare process that allow for consistent implementation regardless of the socioeconomic characteristics of the hospital patient population.

In this study of 131,847 deliveries in 16 hospitals, we expected that hospitals serving higher-income patient populations would report significantly higher rates of bundle compliance than hospitals serving lower-income patient populations. We did not find this relationship; instead none of the relationships we investigated were statistically significant. Where we did see relationships that bordered on statistical significance $(0.064,0.098,0.054$, and 0.138$)$, these were in the opposite direction than we had hypothesized. Therefore, we may have weak evidence that hospitals that serve lower-income populations may actually have better bundle compliance rates. These results were limited by our small sample size. Despite the fact that we did not find any statistically significant results, this does not mean that relationships do not exist; only that future research should investigate these research questions with larger sample sizes.

\subsection{Limitations}

There are several limitations of this study. First, the findings are limited to the hospitals in this project and may not be generalizable to all hospitals. Next, there are many factors that influence bundle compliance rates. Characteristics selected in this study represent a limited set of predictor variables and include broad measures of economic status in a hospital's service area or population, but are not connected with individual patients. Our unit of analysis in this study was the hospital $(\mathrm{N}=16)$. This sample size limits more sophisticated statistical analysis and limits our ability to detect differences between groups. In addition, our study does not include pre-intervention or baseline data or a comparison group. The absence of these measures in our study design limits our ability to utilize alternative analytical methods.

\subsection{Strengths}

This study was strong in the fact that while the overall $\mathrm{N}$ of the study is small, the perinatal safety initiative represented over 131,847 deliveries. The results for this study were consistent across the board for all three poverty measures, indicating that we may indeed be seeing a trend that indicates equality of performance across hospitals. Additionally, this study spans a significant time frame (36 months) which allowed us to measure trends over a large amount of time.

\section{Conclusion}

The findings from this study have important implications for understanding the influence of poverty on care process design. An understanding of Medicaid's role as a resource for pregnant women is key in determining how to improve access to care, assess quality of care, and implement effective and lasting maternity care reform ${ }^{[25]}$. While previous research has indicated that patients of lower socioeconomic status are less likely to receive high quality care, this study does not support those conclusions. Bundle compliance did not vary significantly with regard to Medicaid patient served, median income in the hospital service area, or poverty level in the hospital service area. This indicates that despite economic characteristics of the hospital service area, staff succeeds in implementing high quality evidence based perinatal care bundles. Further research is needed to explore these findings, specifically (1) whether staff at hospitals is consistent in 
bundle compliance regardless of patient economic characteristics, and (2) whether other care measures across these hospitals are administered consistently despite hospital service area economic characteristics.

\section{Acknowledgements}

We would like to acknowledge the Agency for Healthcare Research and Quality for providing funding for this perinatal safety research study. We are grateful to Katy Kozhimannil for her work reviewing and critiquing this paper. Further thanks go to Kristi Miller and Kathy Connolly for their help in data collection.

This project was supported by grant number R18HS019587 from the Agency for Healthcare Research and Quality. The content is solely the responsibility of the authors and does not necessarily represent the official view of the Agency for Healthcare Research and Quality.

\section{References}

[1] AHRQ, Healthcare Cost and Utilization Project. HCUP Facts and Figures Statistics on Hospital-Based Care in the United States [Internet]. 2008. Available from: www.hcup-us.ahrq.gov/reports/factsandfigures/2008/exhibit4_5.jsp. Last accessed July 2011.

[2] Health and Human Services, Centers for Disease Control and Prevention (CDC), National Vital Statistics System. Birth Data [Internet]. CDC. 2011. Available from: www.cdc.gov/nchs/births.htm. Last accessed July 2011.

[3] David RJ, Collins JW Jr. Differing birth weight among infants of U.S.-born Blacks, African-born Blacks, and U.S.-born Whites. N Engl J Med. 1997; 337(17): 1209-1214. PMid:9337381 http://dx.doi.org/10.1056/NEJM199710233371706

[4] Saftlas AF, Koonin LM, Atrash HK. Racial disparity in pregnancy-related mortality associated with livebirth: can established risk factors explain it? Am J Epidemiol. 2000; 152(5): 413-419. PMid:10981453 http://dx.doi.org/10.1093/aje/152.5.413

[5] Amnesty International. Deadly Delivery: The Maternal Health Care Crisis in the USA. London, U.K.:Amnesty International [Internet]. 2010. Available from: http://www.amnestyusa.org/sites/default/files/pdfs/deadlydelivery.pdf. Last accessed July 2011.

[6] Heron M, Hoyert DL, Murphy SL, Xu J, Kochanek KD,Tejada-Vera B. Deaths: Final Data for 2006 [Internet]. Natl Vital Stat Rep. 2009; 57(14): 116, Table 34. Available from: http://www.cdc.gov/nchs/data/nvsr/nvsr57/nvsr57_14.pdf. Last accessed July 2011.

[7] Fiscella K, Franks P, Gold MR, Clancy CM. Inequality in quality: Addressing socioeconomic, racial, and ethnic disparities in health care. JAMA. 2000; 282: 2579-2584. http://dx.doi.org/10.1001/jama.283.19.2579

[8] Kelley E, Moy E, Stryer D. The national healthcare quality and disparities report: An overview. Medical Care. 2005; 43 (3): 13-18.

[9] Fiscella K,Williams D. Health disparities based on socioeconomic inequities: Implications for urban health care. Academic Medicine. 2004; 79: 1139-1147. PMid:15563647 http://dx.doi.org/10.1097/00001888-200412000-00004

[10] Gaylord M, Greer M, Botti J. Improving perinatal health: A novel approach to improve community and adult health. Journal of Perinatatology. 2008; 28: 91-96. PMid:18235508 http://dx.doi.org/10.1038/sj.jp.7211887

[11] Morris A., Hay A., Swann DG, Everingham K., McCulloch C, McNulty J, Walsh TS. Reducing ventilator-associated pneumonia in intensive care: Impact of implementing a care bundle. Critical Care Medicine. 2011; 39(10): 2218-2224. PMid:21666444 http://dx.doi.org/10.1097/CCM.0b013e3182227d52

[12] Miller M, Griswold M, Harris JM, Yenokyan G, Huskins WC, Moss M, Brilli RJ. Decreasing PICU catheter-associated bloodstream infections: NACHRI's quality transformation efforts. Pediatrics. 2010; 125(2): 206-213. PMid:20064860 http://dx.doi.org/10.1542/peds.2009-1382

[13] Riley W, Davis S, Miller K, Hansen H, Sainfort F, Sweet R. Didactic and simulation nontechnical skills team training to improve perinatal patient outcomes in a community hospital. The Joint Commission Journal on Quality and Patient Safety. 2001; 37(8): 357-364.

[14] Schilling L, Dearing JW, Staley P, Harvey P, Fahey L, Kuruppu F. Kaiser Permanente's Performance Improvement System, Part 4: Creating a Learning Organization. The Joint Commission Journal on Quality and Patient Safety. 2011; 36(12): $532-543$.

[15] Cherouny P, Federico F, Haraden C, Leavitt GS, Resar R. Idealized design of perinatal care. IHI innovation series. 2005. Cambridge, MA: Institute for Healthcare Improvement.

[16] Resar RK, Pronovost P, Haraden C, Simmonds T, Rainey T, Nolan T. Using a bundle approach to improve ventilator care process and reduce ventilator associated pneumonia. Joint Commission Journal on Quality and Patient Safety. 2005; 31(5): $243-248$. PMid:15960014

[17] Pronovost PJ, Berenholtz SM, Goeschel CA, Needham DM, Sexton JB, Thompson DA, Hunt E. Creating high reliability in health care organizations. Health Services Research. 2006; 41: 1599-1617. PMid:16898981 http://dx.doi.org/10.1111/j.1475-6773.2006.00567.x 
[18] Knox GE, Simpson KR, Townsend KE. High reliability perinatal units: further observations and a suggested plan for action. ASHRM Journal. 2003; 23: 17-21.

[19] Simpson KR, Knox GE. Strategies for developing an evidence-based approach to perinatal care. American Journal of Maternal and Child Nursing. 2000; 24(3): 122-131. http://dx.doi.org/10.1097/00005721-199905000-00004

[20] DeNavas-Walt C, Proctor BD, Smith JC. Income, poverty, and health insurance coverage in the United States: 2010. U.S. Census Bureau. 2011; 60-239.

[21] Institute for Healthcare Improvement. IHI Perinatal Community Care Bundle Sequencing. IHI [Internet]. 2012. Available from: http://www.ihi.org/knowledge/pages/changes/electiveinductionandaugmentationbundles.aspx. Accessed 11/16/12 online

[22] The Dartmouth Atlas of Healthcare. Data by Region 2011 [Internet]. Available from: at: http://www.dartmouthatlas.org/data/region/ Accessed April 18

[23] Mahlmeister LR. Best practices in perinatal care: Evidence-based management of oxytocin induction and augmentation of labor. Journal of Perinatal \& Neonatal Nursing. 2008; 259-263. PMid:19011488

[24] Clark S, Belfort M, Saade G, Hankins G, Miller D, Frye D, Meyers J. Implementation of a conservative checklist-based protocol for oxytocin administration: maternal and newborn outcomes. American Journal of Obstetrics and Gynecology. 2007; 480e1-480e5.

[25] Monnickendam M, Monnickendam S, Katz C, Katan J. Health Care for the Poor: An Exploration of Primary-care Physicians' Perceptions of Poor Patients and of their Helping Behaviors. Social Science \& Medicine. 2007; 64: 1463-1474. PMid:17234317 http://dx.doi.org/10.1016/j.socscimed.2006.11.033 